\title{
Analysis of synaptotagmin, SV2, and Rab3 expression in cortical glutamatergic and GABAergic axon terminals
}

\author{
Luca Bragina ${ }^{1}$, Giorgia Fattorini ${ }^{1}$, Silvia Giovedi ${ }^{2}$, Marcello Melone ${ }^{1}$, Federica Bosco ${ }^{2}$, Fabio Benfenati ${ }^{2,3}$ \\ and Fiorenzo Conti ${ }^{1,4}$ *
}

\author{
1 Section of Neuroscience and Cell Biology, Department of Experimental and Clinical Medicine, Università Politecnica delle Marche, Ancona, Italy \\ ${ }^{2}$ Department of Experimental Medicine, Università di Genova, Genova, Italy \\ ${ }^{3}$ Department of Neuroscience and Neurotechnologies, The Italian Institute of Technology, Genova, Italy \\ ${ }^{4}$ Fondazione di Medicina Molecolare, Università Politecnica delle Marche, Ancona, Italy
}

\section{Edited by:}

Enrico Cherubini, International School for Advanced Studies, Italy

\section{Reviewed by:}

Dirk Feldmeyer, RWTH Aachen University, Germany

Shaoyu Ge, SUNY Stony Brook, USA

\section{*Correspondence:}

Fiorenzo Conti, Dipartimento di Medicina Sperimentale e Clinica, Università Politecnica delle Marche, Via Tronto 10/A, Torrette di Ancona, I-60020 Ancona, Italy. e-mail:f.conti@univpm.it
We investigated whether cortical glutamatergic and GABAergic release machineries can be differentiated on the basis of the nature and amount of proteins they express, by performing a quantitative analysis of the degree of co-localization of synaptotagmin (SYT) 1 and 2, synaptic vesicle protein 2 (SV2) A and B, and Rab3a and c in VGLUT1+, VGLUT2+, and VGAT+ terminals and synaptic vesicles (SVs) in rat cerebral cortex. Co-localization studies showed that VGLUT1 puncta had high levels of SV2A and B and of Rab3c, intermediate levels of SYT1, and low levels of SYT2 and Rab3c; VGLUT2 puncta exhibited intermediate levels of all presynaptic proteins studied; whereas vesicular GABA transporter (VGAT) puncta had high levels of SV2A and SYT2, intermediate levels of SYT1, Rab3a, and Rab3c, and low levels of SV2B. Since SV2B is reportedly expressed by glutamatergic neurons and we observed SV2B expression in VGAT puncta, we performed electron microscopic studies and found SV2B positive axon terminals forming symmetric synapses. Immunoisolation studies showed that the expression levels of the protein isoforms varied in the three populations of SVs. Expression of SYT1 was highest in VGLUT1-SVs, while SYT2 expression was similar in the three SV groups. Expression of SV2A was similarly high in all three SV populations, except for SV2B levels that were very low in VGAT SVs. Finally, Rab3a levels were similar in the three SV groups, while Rab3c levels were highest in VGLUT1-SVs. These quantitative results extend our previous studies on the differential expression of presynaptic proteins involved in neurotransmitter release in GABAergic and glutamatergic terminals and indicate that heterogeneity of the respective release machineries can be generated by the differential complement of SV proteins involved in distinct stages of the release process.

Keywords: VGAT, VGLUT1, VGLUT2, synaptotagmin, SV2, Rab3

\section{INTRODUCTION}

The possibility that glutamatergic and GABAergic release machineries can be differentiated on the basis of the proteins they express has attracted considerable interest (e.g., Sugino et al., 2006; Micheva et al., 2010). In previous studies, we have approached this question by investigating quantitatively the localization of synapsin I and II (SYNI and II), synaptophysin I and II (SYPI and II), synaptosomal-associated protein (SNAP)-25 and SNAP-23, synaptogyrin (SGYR) 1 and 3, synaptobrevin/vesicle-associated membrane protein (VAMP) 1 and 2, and syntaxin $1 \mathrm{~A}$ and $1 \mathrm{~B}$ (STX1A and B) in vesicular GABA transporter (VGAT)-positive (+) GABAergic and vesicular glutamate transporter VGLUT1+ and VGLUT2+ glutamatergic axon terminals (AT) in cerebral cortex (Bragina et al., 2007, 2010). The results show that the expression of these presynaptic proteins in neocortex varies both between glutamatergic and GABAergic terminals and between VGLUT1+ and VGLUT2+ glutamatergic terminals (Bragina et al., 2007, 2010).

To further define the complement of proteins participating in transmitter release in GABAergic and glutamatergic terminals, we performed a quantitative analysis of the localization of synaptotagmin (SYT) 1 and 2, synaptic vesicle protein 2 (SV2) A and B, and Rab3a and c in VGLUT1+, VGLUT2+, and VGAT+ terminals and synaptic vesicles (SVs) of the cerebral cortex of adult rats. SYT1 and 2 are the main SYT isoforms present in SVs (Sudhof, 2002; Xu et al., 2007). They are known $\mathrm{Ca}^{2+}$ sensors for fast synchronous release and exhibit distinct expression patterns and properties (Geppert et al., 1994b; Fernandez-Chacon et al., 2001; Pang et al., 2006a,b; Xu et al., 2007), and SYT2 appears to be associated to inhibitory neurons and to operate at fast signaling synapses (Geppert et al., 1994b; Pang et al., 2006a,b; Sun et al., 2007). SV2 is a component of all vertebrate SVs (Buckley and Kelly, 1985); it plays a crucial role in the trafficking of SYT to SVs and regulates the effectiveness of calcium in inducing vesicle fusion. SV2A is expressed ubiquitously in the brain, whereas SV2B expression is restricted to forebrain and seems to be lacking in GABAergic neurons (Bajjalieh et al., 1993, 1994; Gronborg et al., 2010). Small GTPases of the Rab family are thought to confer membrane specificity in intracellular fusion reactions (Zerial and McBride, 2001; 
Pfeffer and Aivazian, 2004), and to control release probability, with implications for synaptic plasticity (Schluter et al., 2004, 2006). Rab3a is the most robustly expressed Rab protein in the brain (Geppert et al., 1994a), and an abundant SVs protein (Fischer von Mollard et al., 1990); Rab3c has also been localized to SVs in most brain areas, although at variable levels (Schluter et al., 2002).

Here we report that in the cerebral cortex of adult rats SYT2, SV2B, and Rab3c exhibit significant differences both in the distribution and expression levels between glutamatergic and GABAergic AT and SVs, and that some GABAergic AT express SV2B.

\section{MATERIALS AND METHODS ANIMALS AND TISSUE PREPARATION}

Adult male Sprague-Dawley rats (190-220 g; Charles River, Milan, Italy) were used. All studies were performed in accordance with the E.C. Council Directive 86/609 (November 24, 1986) and were approved by the local authority veterinary service. Animals were kept under a dark-light cycle of $12 \mathrm{~h}$ and permitted food and water ad libitum.

For Western blotting, rats were anesthetized with chloral hydrate (300 mg/kg i.p.) and decapitated. Homogenization of neocortex, membrane preparation, protein determination, and SDSPAGE analysis and immunoblotting were performed as described (Bragina et al., 2006). Precast gels (Tris-HCl; BioRad, Hercules, CA, USA) were used at $15 \%$ polyacrylamide concentration for Rab3 isoforms and at $7.5 \%$ for the remaining proteins. For immunocytochemistry, rats were anesthetized with chloral hydrate (300 mg/kg i.p.), and perfused through the ascending aorta with saline followed by $4 \%$ paraformaldehyde in $0.1 \mathrm{M}$ phosphate buffer (PB; $\mathrm{pH}$ 7.4). Brains were postfixed for $2 \mathrm{~h}$ (immunofluorescence) or $24 \mathrm{~h}$ (electron microscopy) at $4^{\circ} \mathrm{C}$ in the same fixative, cut with a Vibratome into $50 \mu \mathrm{m}$ thick sections, and processed.

\section{ANTIBODIES}

Primary antibodies used in these studies are listed in Table $\mathbf{1 .}$ Western blots were performed to verify antibodies specificity; nitrocellulose filters were probed with antibodies to VGLUT1, VGLUT2, VGAT, SYT1 and 2, SV2A and B, and Rab3a and c at the dilutions reported in Table 1 . After exposure to the appropriate peroxidase-conjugated antibodies (Vector; Burlingame, CA, USA), immunoreactive bands were visualized by BioRad Chemidoc and Quantity One software (BioRad, Hemel Hempstead, UK) using the SuperSignal West Pico (Rockford, IL, USA) chemiluminescent substrate. In cortical crude membrane fractions, all antibodies recognized bands of predicted molecular mass (Figure 1; Matteoli et al., 1991; Bajjalieh et al., 1993; Ullrich et al., 1994; Bellocchio et al., 1998; Chaudhry et al., 1998; Schluter et al., 2002; Varoqui et al., 2002).

\section{CO-LOCALIZATION STUDIES}

Vibratome sections from rat brains were incubated for $1 \mathrm{~h}$ in normal goat serum (NGS; $10 \%$ in $\mathrm{PB}$ ), and overnight at room

Table 1 | Primary antibodies.

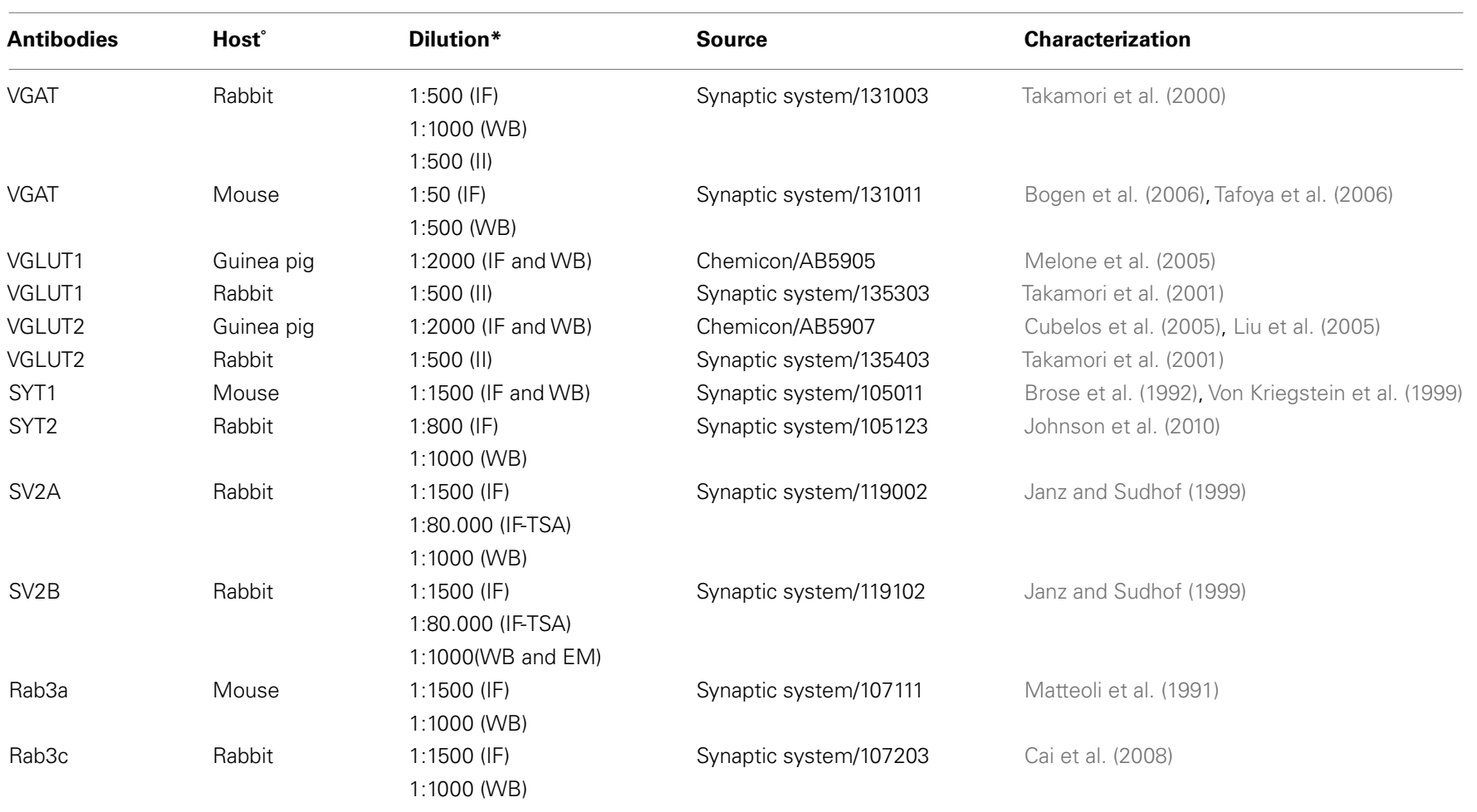

'GP, guinea pig; $M$, mouse; $R$, rabbit; *IF, immunofluorescence; IF-TSA, immunofluorescence with tyramide signal amplification; WB, western blotting; II, immunoisolation; EM, electron microscopy. Western blotting studies for VGLUT1, SYT1, SYT2, SV2A, and SV2B were performed with $5 \mu \mathrm{g}$ of protein, those for VGAT, VGLUT2, Rab3a, and Rab3c with $10 \mu \mathrm{g}$. 
temperature in a solution containing a mixture of the primary antibodies (see Table 2). The next day, sections were incubated in $10 \%$ NGS (30 min) and in a mixture of the appropriate secondary fluorescent antibodies ( $1 \mathrm{~h}$; Table 2). Sections were then mounted, air-dried, and cover slipped using Vectashield mounting medium (H-1000; Vector). For all experimental series (i.e., the vesicular transporters and a presynaptic proteins), the VGLUT1, VGLUT2, and VGAT series were run in parallel to minimize the variability of experimental conditions.

Double-labeled sections were examined using a Leica TCS-SP2 confocal laser microscope equipped with an argon $(488 \mathrm{~nm})$ and a helium/neon $(543 \mathrm{~nm})$ laser for excitation of FITC and TRITC, respectively. Green and red immunofluorescence were imaged sequentially. Control experiments with single-labeled sections and sections incubated either with two primary antibodies and one secondary antibody, or with one primary antibody and two secondary antibodies revealed no appreciable FITC/TRITC bleed-through or antibody cross-reactivity.

Images from all experimental series were from the parietal cortex and were acquired from randomly selected subfields in layers II-VI (at least four to six per layer; two to four sections per animal; 14 rats). Layer I was not sampled because it hardly contains VGAT+ puncta (Chaudhry et al., 1998; Minelli et al., 2003). Images were acquired using a $\times 60$ oil immersion lens (numerical aperture 1.4; pinhole 1.0 and image size $1024 \times 1024$ pixels, yielding a pixel size of $0.06 \mu \mathrm{m}$ ) from a plane in which the resolution of both stains was optimal and never $>1.8 \mu \mathrm{m}$ from the surface (Melone et al., 2005). Signal acquisition was optimized through the " $Q$ LUT" button, which permitted direct visualization of pixel saturation; photomultiplier gain was set so that the brightest pixels were just slightly below saturation, and the offset such that the darkest pixels were just above zero. To avoid bleed-trough between green and red fluorescence, images were acquired sequentially. To improve the signal/noise ratio, 15 frames/image were averaged.

Quantitative analysis was performed in $\sim 8,000$ randomly selected subfields measuring about $25 \mu \mathrm{m} \times 25 \mu \mathrm{m}$ from the $1024 \times 1024$ pixel images. In order to minimize fusion of puncta, contrast of each image was manually adjusted within the maximum range of levels for each color channel. Preliminary studies (not shown) showed that gain/contrast changes within the spectrum used did not alter significantly the percentage of co-localized puncta (Bragina et al., 2010). Then, without reducing the image resolution, each channel was examined separately to identify and manually count immunopositive puncta; the two channels were then merged and the number of co-localizing puncta was counted manually. Puncta were considered double-labeled when overlap was complete or occupied most of the immunopositive puncta and they exhibited morphological similarity (Bragina et al., 2007, 2010; Figure 2).

\section{SYNAPTIC VESICLES IMMUNOISOLATION STUDIES}

Eupergit $\mathrm{C} 1 \mathrm{Z}$ methacrylate microbeads ( $1 \mu \mathrm{m}$ in diameter; Röhm Pharmaceuticals, Darmstadt, Germany) were either blocked with glycine or conjugated with affinity-purified goat anti-rabbit antibodies (IgG; Sigma, Milan, Italy) as previously described (Burger et al., 1991). Affinity-purified anti-VGAT (R), anti-VGLUT1 (R), or anti-VGLUT2 (R) antibodies (Table 1) were conjugated with

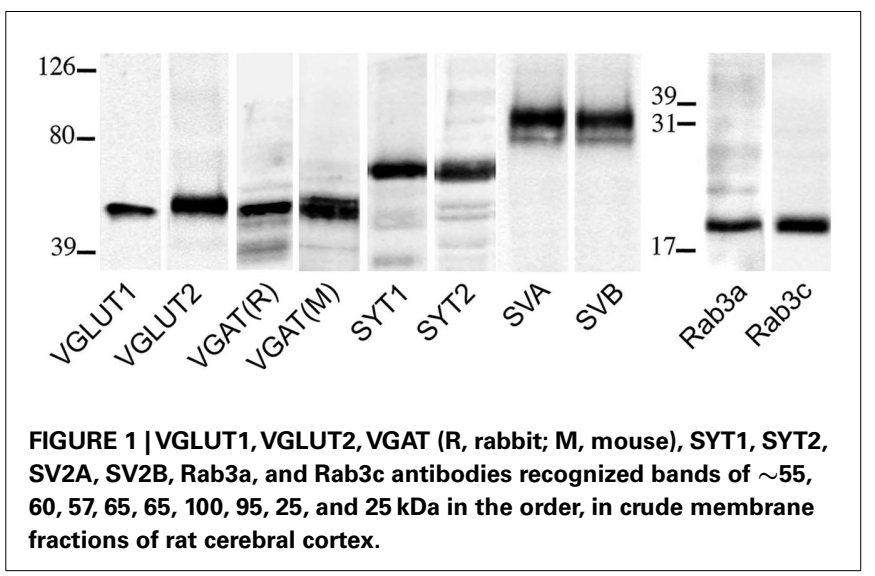

Table 2 | Dilutions of antibodies in double-labeling studies.

\begin{tabular}{|c|c|c|}
\hline Primary antibodies & Secondary antibodies & Dilutions \\
\hline VGLUT1/SYT1 & FITC-GAPA/TRITC-GAMC & $1: 100 / 1: 100$ \\
\hline VGLUT1/SYT2 & FITC-GAPA $/ T R I T C-G A R^{B}$ & $1: 100 / 1: 100$ \\
\hline VGLUT1/SV2A & FITC-GAPA $/ T R I T C-G A R^{B}$ & $1: 100 / 1: 100$ \\
\hline VGLUT1/SV2B & FITC-GAPA/TRITC-GAR ${ }^{B}$ & $1: 100 / 1: 100$ \\
\hline VGLUT1/Rab3a & FITC-GAPA/TRITC-GAM ${ }^{C}$ & $1: 100 / 1: 100$ \\
\hline VGLUT1/Rab3c & FITC-GAPA $/ T R I T C-G A R^{B}$ & $1: 100 / 1: 100$ \\
\hline VGLUT2/SYT1 & FITC-GAPA /TRITC-GAM ${ }^{C}$ & $1: 100 / 1: 100$ \\
\hline VGLUT2/SYT2 & FITC-GAPA/TRITC-GAR ${ }^{B}$ & $1: 100 / 1: 100$ \\
\hline VGLUT2/SV2A & FITC-GAPA/TRITC-GAR ${ }^{B}$ & $1: 100 / 1: 100$ \\
\hline VGLUT2/SV2B & FITC-GAPA/TRITC-GAR ${ }^{B}$ & $1: 100 / 1: 100$ \\
\hline VGLUT2/Rab3a & FITC-GAPA /TRITC-GAM ${ }^{C}$ & $1: 100 / 1: 100$ \\
\hline VGLUT2/Rab3c & FITC-GAPA $/ T R I T C-G A R^{B}$ & $1: 100 / 1: 100$ \\
\hline VGAT/SYT1 & FITC-GAR ${ }^{\mathrm{D}} / \mathrm{TRITC}-\mathrm{GAM}^{\mathrm{C}}$ & $1: 100 / 1: 100$ \\
\hline VGAT/SYT2 & FITC-GAME/TRITC-GAR ${ }^{B}$ & $1: 100 / 1: 100$ \\
\hline VGAT/SV2A & FITC-GAM ${ }^{E} / T R I T C-G A R^{B}$ & $1: 100 / 1: 100$ \\
\hline VGAT/SV2B & FITC-GAM ${ }^{E} / T R I T C-G A R^{B}$ & $1: 100 / 1: 100$ \\
\hline VGAT/Rab3a & FITC-GAR ${ }^{\mathrm{D}} / \mathrm{TRITC}-\mathrm{GAM}^{\mathrm{C}}$ & $1: 100 / 1: 100$ \\
\hline VGAT/Rab3c & FITC-GAME/TRITC-GAR B & $1: 100 / 1: 100$ \\
\hline SYT1/SYT2 & FITC-GAME/TRITC-GAR ${ }^{B}$ & $1: 100 / 1: 100$ \\
\hline SV2A/SV2B & bGAR $(F I T C)^{\mathrm{F}} / \mathrm{TR} I T C-G A R^{\mathrm{B}}$ & $1: 200(1: 50) / 1: 100$ \\
\hline $\mathrm{SV} 2 \mathrm{~B} / \mathrm{SV} 2 \mathrm{~A}$ & bGAR $(\mathrm{FITC})^{\mathrm{F}} / \mathrm{TRITC}-\mathrm{GAR}{ }^{\mathrm{B}}$ & $1: 200(1: 50) / 1: 100$ \\
\hline Rab3a/Rab3c & FITC-GAM ${ }^{E} / T R I T C-G A R^{B}$ & $1: 100 / 1: 100$ \\
\hline
\end{tabular}

${ }^{A}$ Fluorescein isothiocyanate-conjugated goat anti-guinea-pig lgG (Fl-7000, Vector; Burlingame, CA, USA); ${ }^{B}$ tetramethylrhodamine isothiocyanate-conjugated goat anti-rabbit IgG (T-2769, Molecular Probes; Poort Gebouw, The Netherlands); ' tetramethylrhodamine isothiocyanate-conjugated goat anti-mouse $\lg \mathrm{G}$ (T-2762, Molecular Probes); ${ }^{D}$ fluorescein isothiocyanate-conjugated goat antirabbit IgG (FI-1000, Vector); ${ }^{E}$ fluorescein goat anti-mouse IgG (F-2761, Molecular probes): F fluorescein-conjugated tyramide (TSA system).

the covalently bound secondary antibodies, generating IgG-coated anti-VGAT, anti-VGLUT1, or anti-VGLUT2 beads. Beads coated only with glycine or secondary antibodies were used as negative controls (mock beads). Aliquots of the LS1 fraction obtained from osmotic lysis of adult rat cortical synaptosomes (150-300 $\mu \mathrm{g}$ protein/sample; Huttner et al., 1983) was incubated for $2-4 \mathrm{~h}$ at $4^{\circ} \mathrm{C}$ in phosphate-buffered saline (PBS) with the various bead 

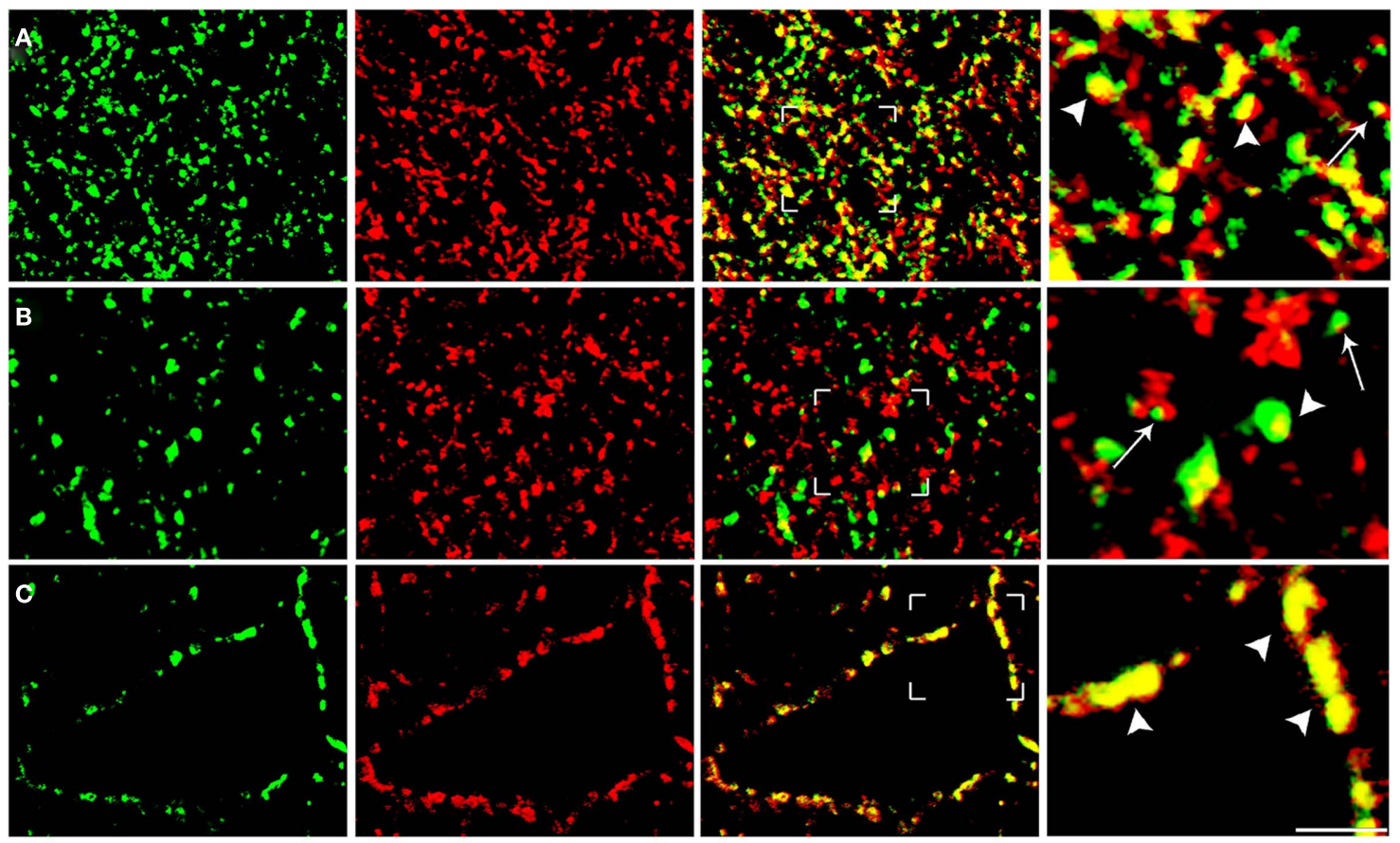

FIGURE 2 |Analysis of confocal microscopy images of cortical sections. The figure shows examples from VGLUT1/SYT1 [row (A)], VGLUT2/Rab3a [row (B)], and VGAT/SV2A series [row (C)]. To minimize the fusion of puncta, the contrast of each image was manually adjusted within the maximum range of levels for each color channel, which was examined separately to identify and count manually immunopositive puncta (first and second columns). Following merging, puncta were considered double-labeled when the overlap was complete or it occupied most of the area of the puncta and they were morphologically similar (arrowheads in fourth columns). Puncta not meeting these criteria (e.g., those indicated by arrows) were not considered double-labeled. Bars: $2 \mu \mathrm{m}$. preparations (50-70 $\mu$ l settled beads in 500 $\mu$ l final volume) under constant rotation. After centrifugation at $1000 \times g$ for $1 \mathrm{~min}$ and repeated washes in PBS, corresponding amounts of bead pellets and supernatant fractions were solubilized in sample buffer and subjected to SDS-PAGE on 7 and $12 \%$ polyacrylamide gels. Gels were electrophoretically transferred to nitrocellulose membranes and immunoblotted with polyclonal or monoclonal SYT1/SYT2, SV2A/SV2B, and Rab3a/Rab3c antibodies (Table 1). Specific immunoreactivity was revealed using peroxidase-conjugated secondary antibodies and the chemiluminescence detection system, as described (Bragina et al., 2007). Quantification of recovered immunoreactivity was performed by densitometric analysis of the fluorograms and by data interpolation into a standard curve of rat brain LS1 fraction run in parallel with the unknown samples. The amounts of synaptic proteins associated with the immunoisolated vesicles were expressed in percent of the respective the total input of LS1 fraction added to the samples. Experiments were repeated at least five times.

\section{ELECTRON MICROSCOPY}

Four sections/rat $(n=2)$ were processed for immunoperoxidase technique and prepared for electron microscopy as described in previous studies (Bragina et al., 2007, 2010). After completion of the immunoperoxidase procedure, sections were flat-embedded in Epon-Spurr (Bragina et al., 2007); then small blocks of tissue containing layer $\mathrm{V}$ were selected by light microscope inspection, glued to blank epoxy, and sectioned with an ultramicrotome. The most superficial ultrathin sections were examined with a Philips EM 208 electron microscope (Eindhoven, The Netherlands) coupled to a MegaView-II high resolution CCD camera (Soft Imaging System; Munster, Germany). Identification of profiles was based on established morphological criteria (Peters et al., 1991).

\section{RESULTS}

The distribution of VGLUT1, VGLUT2, VGAT, SYT1, SYT2, SV2A, SV2B, Rab3a, and Rab3c immunoreactivities was as previously described (Moya et al., 1992; Bajjalieh et al., 1994; Ullrich et al., 1994; Bellocchio et al., 1998; Chaudhry et al., 1998; Kaneko et al., 2002; Minelli et al., 2003; Alonso-Nanclares et al., 2004; Conti et al., 2005). All VGAT+ puncta (e.g., Chaudhry et al., 1998; Minelli et al., 2003) and the vast majority of VGLUT1+ and VGLUT2+ puncta (Kaneko et al., 2002) are AT. Since some astrocytic processes express VGLUT1 or VGLUT2 (Bezzi et al., 2004; Montana et al., 2004) and some of the presynaptic proteins analyzed here (Montana et al., 2006), it is possible that some VGLUT1+ and VGLUT2+ puncta may include rare astrocytic processes. However, considering the paucity of VGLUTs expressed in astrocytes and the elevated number of puncta studied in present studies, the possible bias appears negligible.

We also preliminarily studied the degree of co-localization between pairs of isoforms (SYT1/SYT2, SV2A/SV2B, and $\mathrm{Rab} 3 \mathrm{a} / \mathrm{Rab} 3 \mathrm{c}$ ) in 12 sections from two animals. Co-localization 
was detected in $21.9 \%$ of SYT1 + and $33.4 \%$ of SYT2 + puncta; in $27.6 \%$ of SV2A+ and $48.6 \%$ of SV2B+ puncta; and in $34.1 \%$ of $\mathrm{Rab} 3 \mathrm{a}+$ and $64.2 \%$ of Rab3c+ puncta. Since all pairs of protein isoforms were not highly co-localized, we investigated immunocytochemically whether SYT1 and 2, SV2A and B, Rab3a and c are differentially expressed in VGLUT1+, VGLUT2+, and VGAT+ AT.

\section{EXPRESSION OF SYT1, SYT2, SV2A, SV2B, Rab3a, AND Rab3c IN CORTICAL VGLUT1+, VGLUT2+, AND VGAT+ AXON TERMINALS AND SYNAPTIC VESICLES}

Expression of presynaptic proteins in VGLUT1+ cortical AT was studied in 38 sections from nine rats. Results showed that $60.2 \%$ of VGLUT1 were SYT1+, 36.7\% were SYT2+, 81.7\% SV2A+, 87.7\% $\mathrm{SV} 2 \mathrm{~B}+, 79.1 \% \mathrm{Rab} 3 \mathrm{a}+$, and 20.2\% Rab3c+ (Figure 3; Table 3

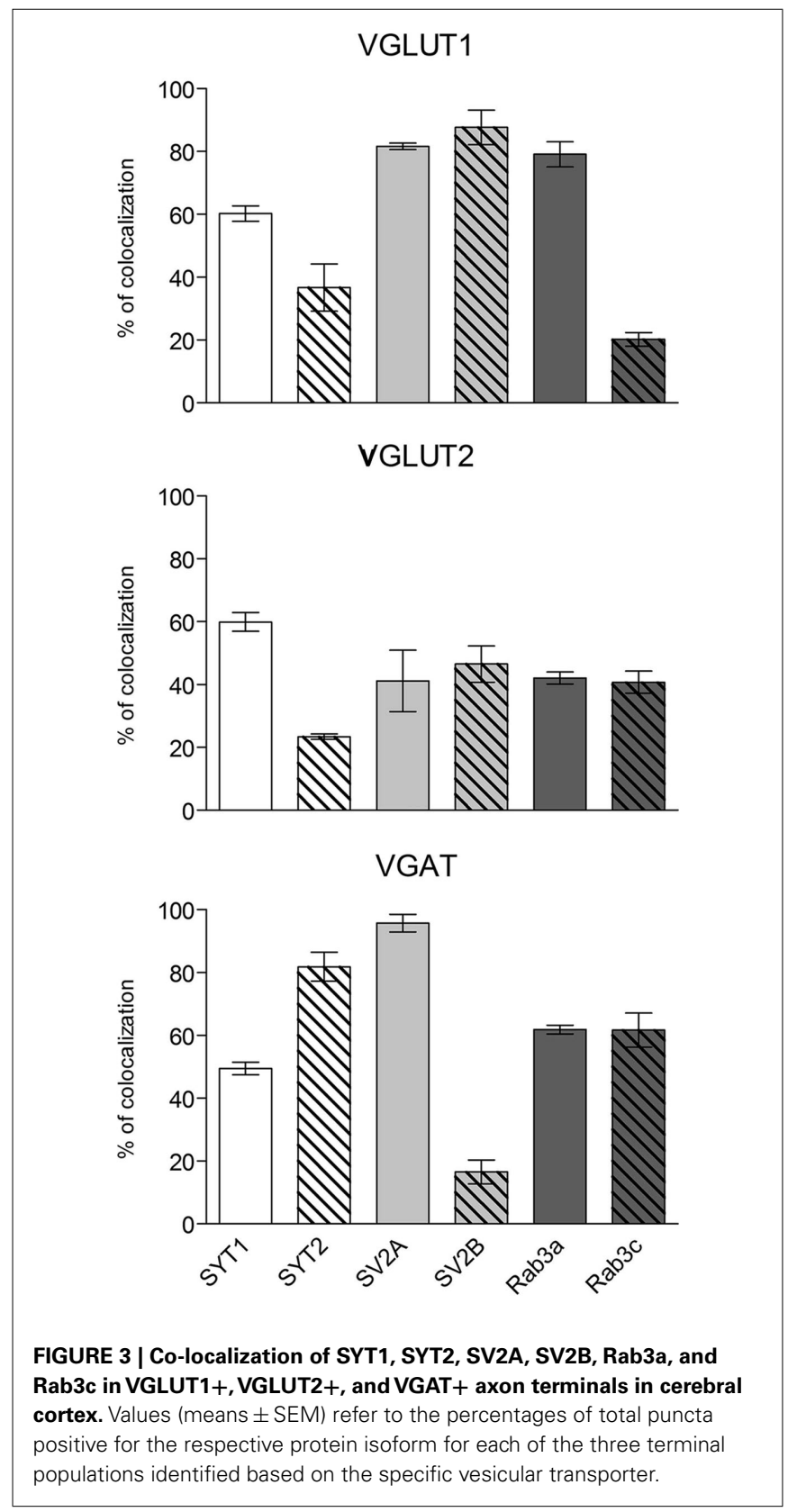

for details). Analysis of VGLUT2 + cortical terminals (40 sections from 10 animals) showed that $59.9 \%$ of them were SYT1+, $23.4 \%$ SYT2+, 41.1\% SV2A+, 46.5\% SV2B+, 42\% Rab3a+, and $40.7 \%$ Rab3c+ (Figure 3; Table 3 for details). Analogous analysis of VGAT + terminals (44 sections from 10 rats) revealed that $49.4 \%$ of them were SYT1+, 81.8\% SYT2+, 95.7\% SV2A+, 16.5\% SV2B+, $61.8 \%$ Rab3a+, and 61.7\% Rab3c+ (Figure 3; Table 3 for details). The degree of co-localization between vesicular transporters and presynaptic proteins studied here did not exhibit any differential laminar distribution.

Previous studies reported that in neocortex SV2B is preferentially expressed at glutamatergic neurons (Bajjalieh et al., 1994). The observation that SV2B was present in some VGAT+ fluorescent terminals was therefore unexpected. To verify whether known GABAergic cortical synapses expressed SV2B, we performed electron microscopy studies in pre-embedded immunoperoxidase material. Our analysis was limited to AT forming symmetric synapses on pyramidal cell bodies in layer $\mathrm{V}$, which are GABAergic and express VGAT (Ribak, 1978; Houser et al., 1984; Chaudhry et al., 1998). Besides confirming that numerous asymmetric synapses are SV2B+, these studies showed that in some cases AT forming perisomatic symmetric synapses were indeed SV2B+ (Figures 4A,B).

These studies provide information on whether a given protein participating in neurotransmitter release is expressed above the threshold for immunocytochemical detection in a subpopulation of identified AT; if this occurs, that terminal is considered to express that protein. However, the percentages reported above result from an all-or-none evaluation of the presence/absence of a given protein in a given terminal and do not provide

Table 3 | SYT1 and 2, SV2A and B, and Rab3a and $c$ in VGLUT1, VGLUT2, and VGAT puncta.

\begin{tabular}{llll}
\hline VT & Puncta (\#) & Co-localization (\%) & PP \\
\hline VGLUT1 & 4640 & $60.2 \pm 2.4$ & SYT1 \\
& 5087 & $36.7 \pm 7.5$ & SYT2 \\
& 6707 & $81.7 \pm 1.0$ & SV2A \\
& 4750 & $87.7 \pm 5.5$ & SV2B \\
& 6351 & $79.1 \pm 4.0$ & Rab3a \\
VGLUT2 & 3770 & $20.2 \pm 2.2$ & Rab3c \\
& 2026 & $59.9 \pm 2.9$ & SYT1 \\
& 3150 & $23.4 \pm 0.8$ & SYT2 \\
& 3254 & $41.1 \pm 9.8$ & SV2A \\
& 2665 & $46.5 \pm 5.8$ & SV2B \\
VGAT & 3345 & $42.0 \pm 1.9$ & Rab3a \\
& 1914 & $40.7 \pm 3.5$ & Rab3c \\
& 1558 & $49.4 \pm 2.0$ & SYT1 \\
& 1758 & $81.8 \pm 4.6$ & SYT2 \\
& 1106 & $95.7 \pm 2.8$ & SV2A \\
& 1793 & $16.5 \pm 3.8$ & SV2B \\
& 1855 & $61.8 \pm 1.4$ & Rab3a \\
& 849 & $61.7 \pm 5.4$ & Rab3c \\
& & &
\end{tabular}

$V T$, vesicular transporter; PP, presynaptic protein. 


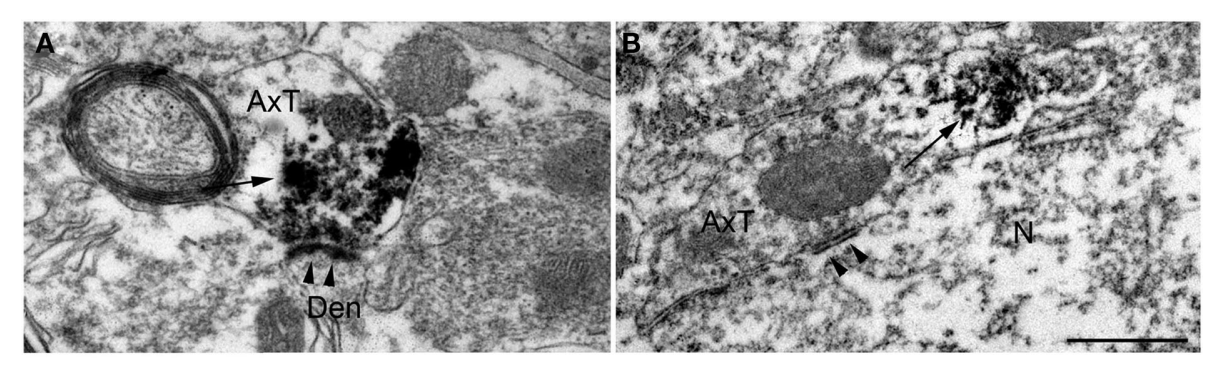

FIGURE 4 | Pre-embedding electron microscopy studies show that SV2B immunoreactivity [arrows in (A,B)] is present in axon terminals forming both asymmetric [arrowheads in (A); layer V] and symmetric [arrowheads in (B); layer V] synaptic contacts. AxT, axon terminal; Den, dendrite; $N$, pyramidal neuron. Scale bar: $500 \mathrm{~nm}$ (A,B).

any information on the expression levels of this protein in glutamatergic and GABAergic SVs. We therefore set up a study to investigate the complement of SYT1, SYT2, SV2A, SV2B, Rab3a, and Rab3c in immunoisolated VGLUT1, VGLUT2, and VGAT $\mathrm{SV}$ populations from the rat neocortex. Immunoisolation studies showed that the expression levels of the protein isoforms varied in the three populations of SVs.

Expression levels of SYT1 was highest in VGLUT1-SVs ( $26.7 \pm 4.3 \%$ of total immunoreactivity), while its levels were lower in VGLUT2-SV (12.9 $\pm 4.3 \%)$ and VGAT SVs $(7.6 \pm 1.9 \%)$, while SYT2 expression was similar in the three SV groups $(27.3 \pm 3.8,30.2 \pm 4.2$, and $23.0 \pm 2.3 \%$ for VGLUT1, VGLUT2, and VGAT SV populations, respectively). Expression levels of SV2A was similarly high in all three SV populations (38.4 \pm 2.4 , $34.8 \pm 4$, and $22.6 \pm 1.1 \%$ for VGLUT1, VGLUT2, and VGAT SVs, respectively), while SV2B levels were similar to those of SV2 in glutamatergic SVs $(45.5 \pm 3.1$ and $28.9 \pm 6.3 \%$ in VGLUT1 and VGLUT2 SVs, respectively), but very low in VGAT SVs $(0.8 \pm 0.43 \%)$. Finally, Rab3a levels were similar in the three SV groups $(4.7 \pm 1.1,3.5 \pm 0.7$, and $3.1 \pm 2.1 \%$ for VGLUT1, VGLUT2, and VGAT SVs, respectively), while Rab3c levels were higher in VGLUT1-SVs $(11.9 \pm 1.4 \%)$ than in VGLUT2 $(6.8 \pm 1.0 \%)$ or VGAT $(3.7 \pm 2.3 \%)$ SVs (Figure 5$)$.

\section{DISCUSSION}

The quantitative studies reported here showed that SYT1/2, $\mathrm{SV} 2 \mathrm{~A} / \mathrm{B}$, and Rab3a/c are differentially expressed in glutamatergic and GABAergic terminals, and that in VGLUT1, VGLUT2, and VGAT terminals the levels of these proteins associated with were also variable.

Under our experimental conditions, SYT1 distribution was similar at glutamatergic and GABAergic terminals, whereas SYT2 expression was more robust in GABAergic terminals. The latter finding extend quantitatively the results of previous studies showing an association between SYT2 and GABAergic neurons (Pang et al., 2006a; Fox and Sanes, 2007). As synapses expressing SYT2 display a faster transmitter release than those expressing SYT1 (Xu et al., 2007), it is conceivable that expression of a given SYT isoform contributes to specific release properties. As far as SV2A and B distribution in the three classes of terminals is concerned, Bajjalieh et al. (1994) and Gronborg et al. (2010) showed that SV2B is preferentially localized to glutamatergic

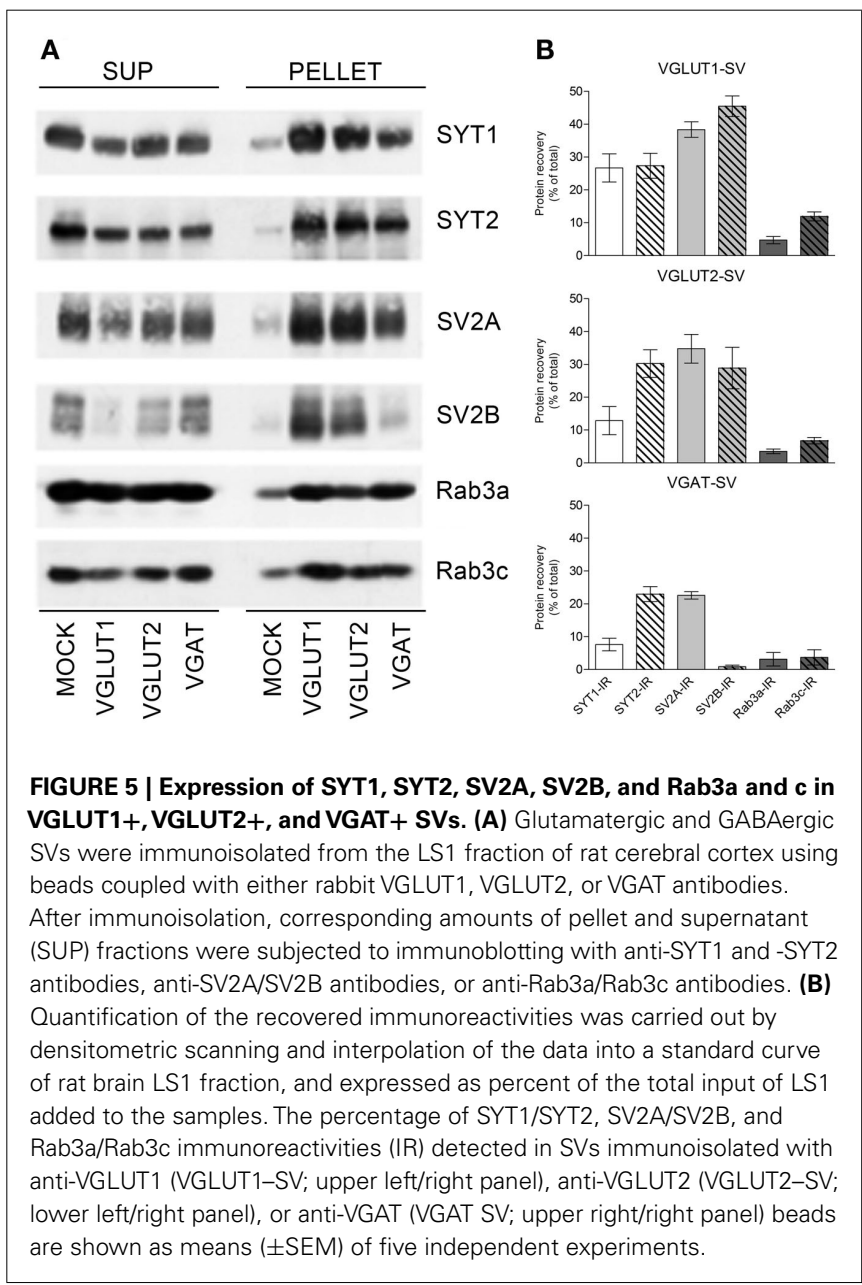

neurons. Our light and electron microscopic immunocytochemical studies add the observation that some VGAT+ terminals express SV2B. However, immunoisolation studies showed very low levels of SV2B in VGAT+ vesicles. The most likely explanation for this discrepancy is presumably correlated to the paucity of SV2B expression in GABAergic terminals. Finally, in our hands Rab3a distribution was more widespread than that of Rab3c in VGLUT1+ terminals, whereas it was similar in VGLUT2 and VGAT+ terminals. 
Besides distribution frequency in the nerve terminal populations, the SV protein isoforms displayed also remarkably different expression levels in VGLUT1, VGLUT2, and VGAT immunoisolated SVs, with SYT2 levels higher than SYT1 levels in VGLUT2 and VGAT SVs and SV2B levels very low compared to SV2A levels in VGAT SVs.

In our previous studies on the heterogeneity of glutamatergic and GABAergic release machinery in neocortex, we demonstrated quantitatively remarkable differences in the expression of presynaptic proteins participating in transmitter release between glutamatergic and GABAergic AT and between VGLUT1 and VGLUT2 glutamatergic terminals, with SYNI, SYNII, SYPI, SNAP-25, and STX1A highly expressed in VGLUT1+ AT, SNAP-23 highly expressed in VGLUT2+ AT and STX1B and VAMP1 more expressed in VGAT+ AT (Bragina et al., 2007, 2010). The present observation that other presynaptic proteins involved in transmitter release exhibit differences between glutamatergic and GABAergic AT, with SV2B preferentially expressed in VGLUT1+ terminals, and SYT2 and Rab3c in VGAT+ ones, strengthens the notion that glutamatergic and GABAergic exocytotic machineries greatly differ in their complement of presynaptic proteins.

Comparison between double-labeling studies, which yields an all-or-none evaluation of expression of a given presynaptic protein in AT, and SV immunoisolation studies, which provide quantitative information on protein expression in homogeneous populations of SVs, allows the definition of a more complete picture of the differential expression of key actors of exocytosis and adds to the issue of molecular heterogeneity of glutamatergic and GABAergic terminals. For example, the VGLUT1 series indicate that in about $50 \%$ of VGLUT $1+$ terminals $\sim 40 \%$ of SVs expressed SYT1 and $\sim 70 \%$ expressed SYT2 (Figure 6), that in most of them about $50 \%$ of SVs expressed SV2A and B, and that in the majority of VGLUT1+ terminals few SVs expressed Rab3a while most of them expressed Rab3c. Thus, the data support the concept that molecular heterogeneity within glutamatergic terminals and between these terminals and GABAergic ones is achieved not only by the presence/absence of a given protein, but also by the relative abundance of SVs expressing a given protein and the number of copies of the protein sorted to each SV (see also Micheva et al., 2010).

In present and previous studies (Bragina et al., 2007, 2010), we reported on the heterogeneous expression of SNARE, calcium sensor, and regulatory proteins at VGLUT1, VGLUT2, and VGAT+ cortical AT. Glutamate- and GABA-operated synapses exhibit different forms of frequency-dependent short-term synaptic plasticity (Galarreta and Hestrin, 1998; Varela et al., 1999; Thomson,

\section{REFERENCES}

Alonso-Nanclares, L., Minelli, A., Melone, M., Edwards, R. H., Defelipe, J., and Conti, F. (2004). Perisomatic glutamatergic axon terminals: a novel feature of cortical synaptology revealed by vesicular glutamate transporter 1 immunostaining. Neuroscience 123, 547-556.

Bajjalieh, S. M., Frantz, G. D., Weimann, J. M., McConnell, S. K., and Scheller, R. H. (1994). Differential expression of synaptic vesicle protein 2

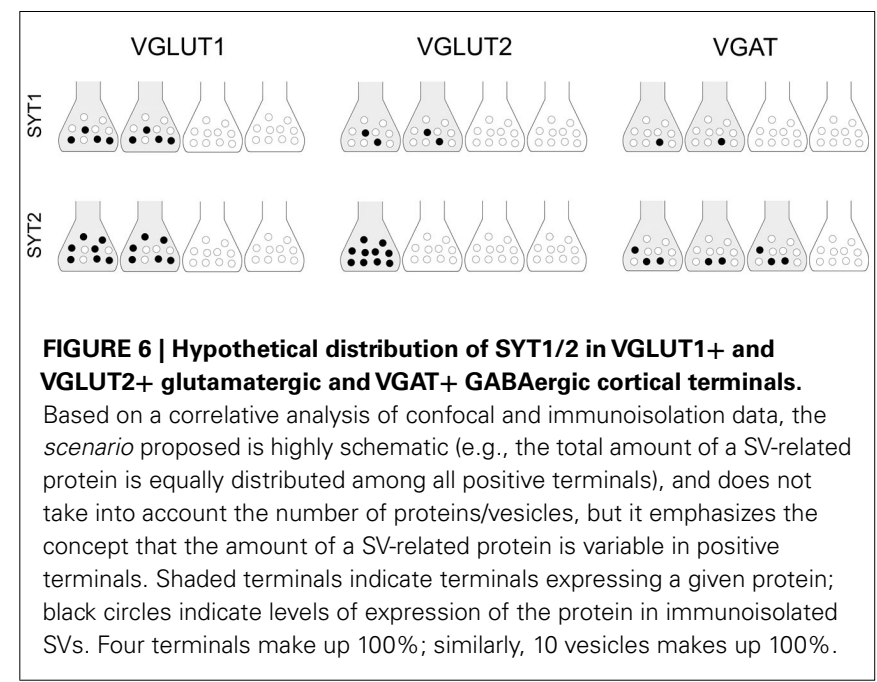

2000), and there is evidence that different isoforms of presynaptic proteins regulate SVs availability at glutamate and GABA synapses (Moulder et al., 2007). Functional differences exist also between VGLUT1 and VGLUT2 terminals: the first ones appear to be associated with low release probability synapses, whereas the latter ones participate in synapses exhibiting higher release probability (Gil et al., 1999; Fremeau et al., 2001). Notwithstanding the known role of other proteins in determining release efficiency (Weston et al., 2011), the present results support the notion that heterogeneous expression levels of presynaptic proteins may contribute to differences in release probability and plasticity (Staple et al., 1997, 2000). Our observation that two types of glutamatergic and GABAergic AT expressed different patterns of presynaptic proteins are in line with this scenario and may contribute to explain functional differences. Whether the different patterns of presynaptic protein expression in the two types of glutamatergic and in GABAergic AT are stable throughout the animal's life or are regulated during development and/or aging will prove a challenge of great interest for future studies.

\section{ACKNOWLEDGMENTS}

This study was supported by research grants from the Italian Ministry of University and Research (PRIN to Fiorenzo Conti, Fabio Benfenati, and Silvia Giovedí), the UNIVPM (to Fiorenzo Conti, Luca Bragina, Giorgia Fattorini, and Marcello Melone), and Telethon-Italy (Grant GGP09134 to Fabio Benfenati).

Bezzi, P., Gundersen, V., Galbete, J. L., Seifert, G., Steinhauser, C., Pilati, E., and Volterra, A. (2004). Astrocytes contain a vesicular compartment that is competent for regulated exocytosis of glutamate. Nat. Neurosci. 7, 613-620.

Bogen, I. L., Boulland, J. L., Mariussen, E., Wright, M. S., Fonnum, F., Kao, H. T., and Walaas, S. I. (2006). Absence of synapsin I and II is accompanied by decreases in vesicular transport of specific neurotransmitters. $J$. Neurochem. 96, 1458-1466.
Bragina, L., Candiracci, C., Barbaresi, P., Giovedi, S., Benfenati, F., and Conti, F. (2007). Heterogeneity of glutamatergic and GABAergic release machinery in cerebral cortex. Neuroscience 146, 1829-1840.

Bragina, L., Giovedi, S., Barbaresi, P., Benfenati, F., and Conti, F. (2010). Heterogeneity of glutamatergic and GABAergic release machinery in cerebral cortex: analysis of synaptogyrin, vesicle-associated membrane protein, and syntaxin. Neuroscience $165,934-943$. 
Bragina, L., Melone, M., Fattorini, G., Torres-Ramos, M., VallejoIllarramendi, A., Matute, C., and Conti, F. (2006). GLT-1 downregulation induced by clozapine in rat frontal cortex is associated with synaptophysin up-regulation. J. Neurochem. 99, 134-141.

Brose, N., Petrenko, A. G., Sudhof, T. C., and Jahn, R. (1992). Synaptotagmin: a calcium sensor on the synaptic vesicle surface. Science 256, 1021-1025.

Buckley, K., and Kelly, R. B. (1985). Identification of a transmembrane glycoprotein specific for secretory vesicles of neural and endocrine cells. J. Cell Biol. 100, 1284-1294.

Burger, P. M., Hell, J., Mehl, E., Krasel, C., Lottspeich, F., and Jahn, R. (1991). GABA and glycine in synaptic vesicles: storage and transport characteristics. Neuron 7, 287-293.

Cai, H., Reim, K., Varoqueaux, F., Tapechum, S., Hill, K., Sorensen, J. B., Brose, N., and Chow, R. H. (2008). Complexin II plays a positive role in $\mathrm{Ca}^{2+}$-triggered exocytosis by facilitating vesicle priming. Proc. Natl. Acad. Sci. U.S.A. 105, 19538-19543.

Chaudhry, F. A., Reimer, R. J., Bellocchio, E. E., Danbolt, N. C., Osen, K. K., Edwards, R. H., and StormMathisen, J. (1998). The vesicular GABA transporter, VGAT, localizes to synaptic vesicles in sets of glycinergic as well as GABAergic neurons. J. Neurosci. 18, 9733-9750.

Conti, F., Candiracci, C., and Fattorini, G. (2005). Heterogeneity of axon terminals expressing VGLUT1 in the cerebral neocortex. Arch. Ital. Biol. 143, 127-132.

Cubelos, B., Gimenez, C., and Zafra, F. (2005). Localization of the GLYT1 glycine transporter at glutamatergic synapses in the rat brain. Cereb. Cortex 15, 448-459.

Fernandez-Chacon, R., Konigstorfer, A., Gerber, S. H., Garcia, J., Matos, M. F., Stevens, C. F., Brose, N., Rizo, J., Rosenmund, C., and Sudhof, T. C. (2001). Synaptotagmin I functions as a calcium regulator of release probability. Nature 410, 41-49.

Fischer von Mollard, G., Mignery, G. A., Baumert, M., Perin, M. S., Hanson, T. J., Burger, P. M., Jahn, R., and Sudhof, T. C. (1990). Rab3 is a small GTP-binding protein exclusively localized to synaptic vesicles. Proc. Natl. Acad. Sci. U.S.A. 87, 1988-1992.

Fox, M. A., and Sanes, J. R. (2007). Synaptotagmin I and II are present in distinct subsets of central synapses. J. Comp. Neurol. 503, 280-296.
Fremeau, R. T. Jr., Troyer, M. D., Pahner, I., Nygaard, G. O., Tran, C. H., Reimer, R. J., Bellocchio, E. E., Fortin, D., Storm-Mathisen, J., and Edwards, R. H. (2001). The expression of vesicular glutamate transporters defines two classes of excitatory synapse. Neuron 31, 247-260.

Galarreta, M., and Hestrin, S. (1998). Frequency-dependent synaptic depression and the balance of excitation and inhibition in the neocortex. Nat. Neurosci. 1, 587-594.

Geppert, M., Bolshakov, V. Y., Siegelbaum, S. A., Takei, K., De Camilli, P., Hammer, R. E., and Sudhof, T. C. (1994a). The role of Rab3A in neurotransmitter release. Nature 369, 493-497.

Geppert, M., Goda, Y., Hammer, R. E., Li, C., Rosahl, T. W., Stevens, C. F., and Sudhof, T. C. (1994b). Synaptotagmin I: a major $\mathrm{Ca}^{2+}$ sensor for transmitter release at a central synapse. Cell 79, 717-727.

Gil, Z., Connors, B. W., and Amitai, Y. (1999). Efficacy of thalamocortical and intracortical synaptic connections: quanta, innervation, and reliability. Neuron 23, 385-397.

Gronborg, M., Pavlos, N. J., Brunk, I., Chua, J. J., Munster-Wandowski, A., Riedel, D., Ahnert-Hilger, G., Urlaub, H., and Jahn, R. (2010). Quantitative comparison of glutamatergic and GABAergic synaptic vesicles unveils selectivity for few proteins including MAL2, a novel synaptic vesicle protein. J. Neurosci. 30, 2-12.

Houser, C. R., Vaughn, D. E., Hendry, S. H., Jones, E. G., and Peters, A. (1984). "GABA neurons in the cerebral cortex," in Cerebral Cortex, eds E. G. Jones and A. Peters (New York: Plenum Press), 63-89.

Huttner, W. B., Schiebler, W., Greengard, P., and De Camilli, P. (1983). Synapsin I (protein I), a nerve terminal-specific phosphoprotein. III. Its association with synaptic vesicles studied in a highly purified synaptic vesicle preparation. J. Cell Biol. 96, 1374-1388.

Janz, R., and Sudhof, T. C. (1999). SV2C is a synaptic vesicle protein with an unusually restricted localization: anatomy of a synaptic vesicle protein family. Neuroscience 94, 1279-1290.

Johnson, S. L., Franz, C., Kuhn, S., Furness, D. N., Ruttiger, L., Munkner, S., Rivolta, M. N., Seward, E. P., Herschman, H. R., Engel, J., Knipper, M., and Marcotti, W. (2010). Synaptotagmin IV determines the linear $\mathrm{Ca}^{2+}$ dependence of vesicle fusion at auditory ribbon synapses. Nat. Neurosci. 13, 45-52.
Kaneko, T., Fujiyama, F., and Hioki, H. (2002). Immunohistochemical localization of candidates for vesicular glutamate transporters in the rat brain. J. Comp. Neurol. 444, 39-62.

Liu, X. B., Low, L. K., Jones, E. G., and Cheng, H. J. (2005). Stereotyped axon pruning via plexin signaling is associated with synaptic complex elimination in the hippocampus. $J$. Neurosci. 25, 9124-9134.

Matteoli, M., Takei, K., Cameron, R., Hurlbut, P., Johnston, P. A., Sudhof, T. C., Jahn, R., and De Camilli, P. (1991). Association of Rab3A with synaptic vesicles at late stages of the secretory pathway. J. Cell Biol. 115, 625-633.

Melone, M., Burette, A., and Weinberg, R. J. (2005). Light microscopic identification and immunocytochemical characterization of glutamatergic synapses in brain sections. J. Comp. Neurol. 492, 495-509.

Micheva, K. D., Busse, B., Weiler, N. C., O'Rourke, N., and Smith, S. J. (2010). Single-synapse analysis of a diverse synapse population: proteomic imaging methods and markers. Neuron 68, 639-653.

Minelli, A., Alonso-Nanclares, L. Edwards, R. H., DeFelipe, J., and Conti, F. (2003). Postnatal development of the vesicular GABA transporter in rat cerebral cortex. Neuroscience 117, 337-346.

Montana, V., Malarkey, E. B., Verderio, C., Matteoli, M., and Parpura, V. (2006). Vesicular transmitter release from astrocytes. Glia 54, 700-715.

Montana, V., Ni, Y., Sunjara, V., Hua, X., and Parpura, V. (2004). Vesicular glutamate transporter-dependent glutamate release from astrocytes. $J$. Neurosci. 24, 2633-2642.

Moulder, K. L., Jiang, X., Taylor, A. A., Shin, W., Gillis, K. D., and Mennerick, S. (2007). Vesicle pool heterogeneity at hippocampal glutamate and GABA synapses. J. Neurosci. 27, 9846-9854.

Moya, K. L., Tavitian, B., Zahraoui, A., and Tavitian, A. (1992). Localization of the ras-like rab3A protein in the adult rat brain. Brain Res. 590, 118-127.

Pang, Z. P., Melicoff, E., Padgett, D., Liu, Y., Teich, A. F., Dickey, B. F., Lin, W., Adachi, R., and Sudhof, T. C. (2006a). Synaptotagmin-2 is essential for survival and contributes to $\mathrm{Ca}^{2+}$ triggering of neurotransmitter release in central and neuromuscular synapses. J. Neurosci. 26, 13493-13504.

Pang, Z. P., Shin, O. H., Meyer, A. C. Rosenmund, C., and Sudhof, T. C. (2006b). A gain-of-function mutation in synaptotagmin-1 reveals a critical role of $\mathrm{Ca}^{2+}$-dependent soluble N-ethylmaleimide-sensitive factor attachment protein receptor complex binding in synaptic exocytosis. J. Neurosci. 26, 12556-12565.

Peters, A., Palay, S. L., and Webster, H. (1991). The Fine Structure of the Nervous System: Neurons and their Supporting Cells. New York: Oxford University Press.

Pfeffer, S., and Aivazian, D. (2004). Targeting Rab GTPases to distinct membrane compartments. Nat. Rev. Mol. Cell Biol. 5, 886-896.

Ribak, C. E. (1978). Aspinous and sparsely-spinous stellate neurons in the visual cortex of rats contain glutamic acid decarboxylase. J. Neurocytol. 7, 461-478.

Schluter, O. M., Basu, J., Sudhof, T. C., and Rosenmund, C. (2006). Rab3 superprimes synaptic vesicles for release: implications for short-term synaptic plasticity. J. Neurosci. 26, 1239-1246.

Schluter, O. M., Khvotchev, M., Jahn, R., and Sudhof, T. C. (2002). Localization versus function of Rab3 proteins. Evidence for a common regulatory role in controlling fusion. $J$. Biol. Chem. 277, 40919-40929.

Schluter, O. M., Schmitz, F., Jahn, R., Rosenmund, C., and Sudhof, T. C. (2004). A complete genetic analysis of neuronal Rab3 function. $J$. Neurosci. 24, 6629-6637.

Staple, J. K., Morgenthaler, F., and Catsicas, S. (2000). Presynaptic heterogeneity: Vive la difference. News Physiol. Sci. 15, 45-49.

Staple, J. K., Osen-Sand, A., Benfenati, F., Pich, E. M., and Catsicas, S. (1997). Molecular and functional diversity at synapses of individual neurons in vitro. Eur. J. Neurosci. 9, 721-731.

Sudhof, T. C. (2002). Synaptotagmins: why so many? J. Biol. Chem. 277, 7629-7632.

Sugino, K., Hempel, C. M., Miller, M. N., Hattox, A. M., Shapiro, P., Wu, C., Huang, Z. J., and Nelson, S. B. (2006). Molecular taxonomy of major neuronal classes in the adult mouse forebrain. Nat. Neurosci. 9, 99-107.

Sun, J., Pang, Z. P., Qin, D., Fahim, A. T., Adachi, R., and Sudhof, T. C. (2007). A dual-Ca ${ }^{2+}$-sensor model for neurotransmitter release in a central synapse. Nature 450, 676-682.

Tafoya, L. C., Mameli, M., Miyashita, T., Guzowski, J. F., Valenzuela, C. F., and Wilson, M. C. (2006). Expression and function of SNAP-25 as a universal SNARE component in 
Bragina et al.

Heterogeneity of cortical axon terminals

GABAergic neurons. J. Neurosci. 26, 7826-7838.

Takamori, S., Rhee, J. S., Rosenmund, C., and Jahn, R. (2001). Identification of differentiation-associated brainspecific phosphate transporter as a second vesicular glutamate transporter (VGLUT2). J. Neurosci. 21, RC182.

Takamori, S., Riedel, D., and Jahn, R. (2000). Immunoisolation of GABAspecific synaptic vesicles defines a functionally distinct subset of synaptic vesicles. J. Neurosci. 20, 4904-4911.

Thomson, A. M. (2000). Molecular frequency filters at central synapses. Prog. Neurobiol. 62, 159-196.

Ullrich, B., Li, C, Zhang, J. Z., McMahon, H., Anderson, R. G., Geppert, M., and Sudhof, T. C. (1994). Functional properties of multiple synaptotagmins in brain. Neuron 13, Weston, M. C., Nehring, R. B., Woj1281-1291. cik, S. M., and Rosenmund, C.

Varela, J. A., Song, S., Turrigiano, G. (2011). Interplay between VGLUT G., and Nelson, S. B. (1999). Dif- isoforms and endophilin Al reguferential depression at excitatory and lates neurotransmitter release and inhibitory synapses in visual cortex. short-term plasticity. Neuron 69, J. Neurosci. 19, 4293-4304.

Varoqui, H., Schafer, M. K., Zhu, H., Xu, J., Mashimo, T., and Sudhof, T. C. Weihe, E., and Erickson, J. D. (2002). $\quad$ (2007). Synaptotagmin-1, -2, and -9: Identification of the differentiation- $\quad \mathrm{Ca}(2+)$ sensors for fast release that associated $\mathrm{Na}+/ \mathrm{PI}$ transporter as specify distinct presynaptic propera novel vesicular glutamate trans- ties in subsets of neurons. Neuron 54, porter expressed in a distinct set of glutamatergic synapses. J. Neurosci. 22, 142-155.

Von Kriegstein, K., Schmitz, F., Link, E., and Sudhof, T. C. (1999). Distribution of synaptic vesicle proteins in the mammalian retina identifies obligatory and facultative components of ribbon synapses. Eur. J. Neurosci. 11, 1335-1348. could be construed as a potential conflict of interest.

Received: 08 November 2011; accepted: 25 December 2011; published online: 10 January 2012.

Citation: Bragina L, Fattorini G, Giovedí $S$, Melone M, Bosco F, Benfenati F and Conti F (2012) Analysis of synaptotagmin, SV2, and Rab3 expression in cortical glutamatergic and GABAergic axon terminals. Front. Cell. Neurosci. 5:32. doi: 10.3389/fncel.2011.00032

Copyright (C) 2012 Bragina, Fattorini, Giovedi, Melone, Bosco, Benfenati and Conti. This is an open-access article distributed under the terms of the Creative Commons Attribution Non Commercial License, which permits noncommercial use, distribution, and reproduction in other forums, provided the original authors and source are credited.

Frontiers in Cellular Neuroscience

www.frontiersin.org

January 2012 | Volume 5 | Article 32 | 9 